\title{
Simvastatin reverses cardiomyocyte hypertrophy via the upregulation of phosphatase and tensin homolog expression
}

\author{
YONG-QING CHEN ${ }^{1}$, LIAN-YOU ZHAO ${ }^{2}$, WEI-ZE ZHANG ${ }^{1}$ and TAO LI ${ }^{1}$ \\ ${ }^{1}$ Department of Cardiology, General Hospital of Lanzhou Military Area Command, \\ Lanzhou, Gansu 730050; ${ }^{2}$ Department of Cardiology, Tangdu Hospital, \\ Fourth Military Medical University, Xi'an, Shaanxi 710038, P.R. China
}

Received August 12, 2014; Accepted May 1, 2015

DOI: $10.3892 /$ etm.2015.2550

\begin{abstract}
The aim of the present study was to investigate the effects of simvastatin on the protein kinase B (PKB) signaling pathway and the expression of phosphatase and tensin homolog (PTEN). The effects of simvastatin were analyzed by administering the drug orally to male spontaneously hypertensive rats (SHRs) at a dose of $10 \mathrm{mg} / \mathrm{kg} / \mathrm{day}$, while the control animals received an equal volume of saline. The systolic pressure $(\mathrm{mmHg})$ of the rat tail artery was measured prior to the initiation of the experiment, and once a week until the end of the experiment. At the end of the experiment, the animals were euthanized and the hearts were removed. The left ventricular and interventricular septum were weighed, after which the left ventricular mass/body mass ratio was calculated. In addition, cardiomyocytes isolated from Sprague Dawley rats were cultured with $15 \%$ fetal bovine serum to induce hypertrophy, following which the cells were treated with different doses of simvastatin. The in vitro effects were assessed by measuring the surface area of the cardiomyocytes, while the rate of protein synthesis was measured using a $3 \mathrm{H}$-leucine incorporation assay and western blot analyses. Simvastatin was demonstrated to inhibit cardiomyocyte hypertrophy in the in vivo and in vitro experiments. Notably, simvastatin increased PTEN expression and inhibited PKB expression in the SHR model, as well as in the cardiomyocytes in culture. In addition, the use of PTEN antisense oligodeoxynucleotides was revealed to inhibit the effects of simvastatin on cardiomyocytes. Therefore, these results indicated that simvastatin was able to reverse cardiomyocyte hypertrophy in vivo and in vitro, possibly by increasing the expression of PTEN.
\end{abstract}

Correspondence to: Dr Yong-Qing Chen, Department of Cardiology, General Hospital of Lanzhou Military Area Command, 333 Middle Nanbinhe Road, Lanzhou, Gansu 730050, P.R. China

E-mail: chyqmd@126.com

Key words: simvastatin, protein kinase B, phosphatase and tensin homolog, cardiomyocyte hypertrophy

\section{Introduction}

Hypertrophy of cardiomyocytes pathologically contributes to left ventricular hypertrophy (LVH), which is induced by hypertension (1). Thus, elucidation of the mechanisms underlying the development and regulation of cardiomyocyte hypertrophy is of great importance in the prevention of cardiovascular diseases. Statins, or 3-hydroxy-3-methylglutaryl coenzyme A reductase inhibitors, have been reported to exert multiple protective effects on the cardiovascular system, independent of their classical functions on lipoproteins. There is accumulating evidence that statins, in addition to their classical functions on lipoproteins, also reduce the level of cellular isoprenoid intermediates and inhibit the isoprenylation of small molecular weight G-proteins of the Ras superfamily (Ras/Rho), to subsequently exert protective effects on the cardiovascular system (2). Statins have been demonstrated to exert their effects on cell proliferation and hypertrophy through mediating the membrane translocation of Ras protein. The mitogen-activated protein kinase (MAPK) signaling pathway and the phosphatidylinositol 3-kinase (PI3K)/protein kinase B (PKB) signaling pathway are known to be involved in the biological effects of Ras protein (3). In addition, the PI3K/PKB pathway has been demonstrated to be closely involved in the proliferation, hypertrophy, apoptosis and survival process of cells (4).

The cellular kinase, PKB, is a serine/threonine protein kinase that is activated via the PI3K signaling pathway. As a common hub in a number of antiapoptotic pathways, PKB functions through the phosphorylation of a plethora of downstream targets, which in turn affect protective homeostatic, apoptotic and necrotic pathways, as well as a number of other pathways, including those associated with growth and development, and glucose metabolism (5). PKB is an important mediator of PI3K signaling, and numerous studies have implicated PKB-dependent signaling pathways in the regulation of cardiac growth, contractile function and coronary angiogenesis $(6,7)$.

Phosphatase and tensin homolog (PTEN) is a lipid phosphatase that predominantly dephosphorylates phosphatidylinositol 3,4,5-trisphosphate [PtdIns-(3,4,5)-P3] to generate PtdIns-(4,5)-P2, and therefore functions as a PI3K/PKB antagonist (8). In Drosophila, the loss of PTEN has been 
shown to result in enhanced growth, whereas PTEN overexpression has been demonstrated to decrease the cell number and cell size (9). Furthermore, in mice, the deletion of PTEN in cardiac muscle cells has been shown to result in cardiac hypertrophy, which is associated with an increase in individual myocyte size and PKB activity (10). These results are consistent with the hypothesis that the loss of PTEN results in increased PI3K/PKB activity, leading to cardiac hypertrophy. However, whether statins exert an effect on the hypertrophy of cardiomyocytes remains unknown, and the specific biological mechanism remains poorly understood.

In the present study, the effects of simvastatin were investigated on spontaneously hypertensive rats (SHRs) in vivo and cardiomyocytes with serum-induced hypertrophy in vitro. Subsequently, the effects of simvastatin on PKB and PTEN expression in vivo and in vitro were investigated. In addition, the effects of applying PTEN antisense oligodeoxynucleotides on the biological effects of simvastatin in cardiac myocytes were assessed in order to elucidate the function of PTEN relative to the PKB pathway. Therefore, the aim of the present study was to provide insight into the molecular mechanisms modulating the hypertrophy of cardiomyocytes, and identify potential therapeutic targets for the prevention of LVH induced by hypertension.

\section{Materials and methods}

Animals. Thirty male SHRs and 15 Wistar-Kyoto (WKY) rats (age, 8 weeks) were obtained from the Animal Center of Fuwai Hospital (Beijing, China). The rats had free access to water and a regular diet, and were housed at $22 \pm 1^{\circ} \mathrm{C}$ under a standard 12-h light/dark cycle to acclimate for one week prior to the experiments. All animals in the study were cared for, and the experiments were performed according to, the established Guide for the Care and Use of Laboratory Animals published by the National Institutes of Health (NIH Publication No. 85-23, revised 1996). The study was approved by the Institutional Review Board and the Animal Care and Use Committee of the General Hospital of Lanzhou Military Area Command (Lanzhou, China).

Determination of the rat tail artery systolic pressure. The systolic pressure $(\mathrm{mmHg})$ of the rat tail artery was measured using a rat sphygmomanometer (RBP-1B-type, China Japan Friendship Institute of Clinical Medicine, Beijing, China). All measurements for the rat tail artery systolic pressure were performed by the same investigator. The blood pressure of each animal was measured prior to the initiation of the experiment, and once a week until the end of the experiment.

Simvastatin administration for in vivo study. Simvastatin (Wuhan C-bons Group, Wuhan, China) has been widely administered clinically to prevent cardiovascular and cerebrovascular diseases (11). Each animal received simvastatin $(10 \mathrm{mg} / \mathrm{kg})$ once a day over a 10-week study period. Control animals received the same volume of saline during the same schedule.

Calculation of the left ventricular mass (LVM) to body mass $(B M)$ ratio. Following weighing, the animals were anesthetized with an intraperitoneal injection of $3 \%$ pentobarbital sodium $(40 \mathrm{mg} / \mathrm{kg})$. The rat was sacrificed by exsanguination whilst still under anaesthesia. The heart was removed immediately following euthanasia and rinsed with $0.9 \%$ saline $\left(4^{\circ} \mathrm{C}\right)$. Subsequently, the vessels, cardiac atrium and right ventricular were quickly removed. After blotting the saline with filter paper, the left ventricular and interventricular septum were weighed, from which the LVM/BM ratio was calculated. Finally, the tissue samples were cut into sections at $6-\mu \mathrm{m}$ thickness with an interval of $300 \mu \mathrm{m}$ between each section and stored in liquid nitrogen until required.

Culture and identification of the cardiomyocytes. Cardiomyocytes were cultured and identified according to a previously reported method with slight modifications (12). Briefly, the ventricles of the Sprague Dawley rats (age, 1-3 days; either gender; Research Center of Experimental Animals in the Fourth Military Medical University, Xi'an, China) were harvested aseptically and cut into $1-\mathrm{mm}^{3}$ pieces. The tissue samples were digested with $0.1 \%$ type I collagenase (Sigma-Aldrich, St. Louis, MO, USA) at $37^{\circ} \mathrm{C}$ for $5-10 \mathrm{~min}$ with the assistance of magnetic stirring. The digestion procedures were repeated 5-7 times. Following centrifugation at $550 \mathrm{x}$ g for $5 \mathrm{~min}$ at $37^{\circ} \mathrm{C}$, the cell pellets were collected and suspended in Dulbecco's modified Eagle's medium (DMEM; Gibco Life Technologies, Grand Island, NY, USA) containing 15\% neonatal bovine serum (Hangzhou Sijiqing Biological Engineering Materials Co., Ltd., Hangzhou, China). The cells were seeded into culture flasks and allowed to stand for $2 \mathrm{~h}$ at $37^{\circ} \mathrm{C}$ in $5 \%$ $\mathrm{CO}_{2}\left(\mathrm{CO}_{2}\right.$ incubator; NuAire, Hong Kong, China). After $2 \mathrm{~h}$, the difference in the adhesion ability of the cells enabled the isolation of the cardiomyocytes. The isolated cardiomyocytes were seeded into culture flasks or plates at a density of $4 \times 10^{5}$ cells $/ \mathrm{ml}$. After 48 h, $0.1 \mathrm{mmol} / 1$ bromodeoxyuridine (Sigma-Aldrich) was added to inhibit the growth of the cells other than the cardiomyocytes. The medium was changed every 2 days. The cells were identified as cardiomyocytes by spontaneous beating after $24 \mathrm{~h}$ and positive staining for $\alpha$-smooth muscle actin. The staining procedure is shown as previously described (13). The purity of the cells reached $95 \%$, which was satisfactory for the further experiments.

Intervention protocol. Stably cultured cells were divided into three groups. In the control group, the cells were cultured with serum free DMEM, while in the serum group, the cells were cultured with DMEM containing $15 \%$ neonatal bovine serum. In the simvastatin intervention group, the cells were cultured with DMEM containing $15 \%$ neonatal bovine serum, in addition to various concentrations of simvastatin $\left(10^{-5}, 10^{-6}, 10^{-7}\right.$ and $\left.10^{-8} \mathrm{~mol} / \mathrm{l}\right)$. After $24 \mathrm{~h}$, cell cultivation was terminated for further evaluation of the indices.

Determination of the cardiomyocyte surface area. Cardiomyocytes were seeded at a density of $4 \times 10^{5}$ cells $/ \mathrm{ml}$ into six-well plates, with a cover slip placed previously. The cells were subsequently cultured with medium containing a variety of intervention factors. After $24 \mathrm{~h}$ of culture, the cultivation was terminated and the cells on the cover slip were fixed with $95 \%$ alcohol for $15 \mathrm{~min}$ in preparation for hematoxylin and eosin staining. A Leica-Q500 image analysis system (Leica Camera AG, Wetzlar, Germany) was used to 
determine the cardiomyocyte surface area. Five observation fields were selected randomly on each cover slip, and 5-8 cells within each observation field were selected for the determination of the mean cardiomyocyte surface area according to the image analysis system. The average of the measurements was calculated.

Determination of the protein synthesis rate using a 3 H-leucine incorporation assay. Cardiomyocytes were seeded into 24 -well plates at a density of $4 \times 10^{5}$ cells $/ \mathrm{ml}$, with $1 \mathrm{ml}$ in each well. Next, $3 \mathrm{H}$-leucine, with a final concentration of $3.7 \times 10^{4} \mathrm{~Bq} / \mathrm{ml}$ (China Institute of Atomic Energy, Beijing, China) that had been prewarmed to $37^{\circ} \mathrm{C}$, was added to each well together with the intervention factors. Following termination of the cultivation, the cells were washed twice with phosphate-buffered saline (PBS) and 10\% trichloroacetic acid for $5 \mathrm{~min}$, which had both been precooled to $4^{\circ} \mathrm{C}$. Subsequently, $0.5 \mathrm{ml} \mathrm{NaOH}-1 \% \operatorname{SDS}(0.3 \mathrm{~mol} / \mathrm{l})$ was added, and the mixture was allowed to stand for $30 \mathrm{~min}$ at room temperature. The cell lysates were harvested, transferred to glass-fiber filter paper, and subjected to drying at $42^{\circ} \mathrm{C}$. The radioactivity (cpm/cell) of the cells was evaluated using a LS6500 liquid scintillation counter (Beckman Coulter, Brea, CA, USA).

Western blot analysis. After $48 \mathrm{~h}$ cultivation, the cells were washed with cold PBS, and lysed with $100 \mu \mathrm{l} 1 \mathrm{X}$ SDS lysis buffer, which had been prewarmed to $85^{\circ} \mathrm{C}$. The lysates were collected in a microtube, heated for $10 \mathrm{~min}$, and then centrifuged at $12,000 \mathrm{x} \mathrm{g}$ for $10 \mathrm{~min}$ at room temperature. Following collection of the supernatant, the protein concentration was determined according to the Bradford method (Bio-Rad Laboratories, Inc., Hercules, CA, USA). Subsequently, $20 \mu \mathrm{g}$ protein from each sample was boiled at $100^{\circ} \mathrm{C}$ for $5 \mathrm{~min}$, after which the proteins were separated through 5-8\% SDS-PAGE. The protein band was transferred to a nitrocellulose membrane, and the membranes were blocked with $3 \%$ bovine serum albumin solution overnight at $4^{\circ} \mathrm{C}$. The detection of the different proteins was conducted by overnight incubation of the membrane at $4^{\circ} \mathrm{C}$ with the required dilution of a specific primary antibody (rabbit anti-mouse PKB monoclonal antibody; cat. no. SC-7686; dilution, 1:200; Santa Cruz Biotechnology, Inc., Santa Cruz, CA, USA; rabbit anti-mouse PTEN monoclonal antibody; dilution, 1:500; Sigma-Aldrich). The membrane was further incubated with a horseradish peroxidase-coupled goat anti-rabbit IgG (cat. no. sc-2004; Santa Cruz Biotechnology, Inc.) for $1 \mathrm{~h}$. After thoroughly washing with $0.05 \%$ Tween 20 , the protein bands were visualized using 3,3'-diaminobenzidine (Sigma-Aldrich). LabWorks 3.0 UVP software (UVP, Upland, CA, USA) was used to analyze the optical density, which represented the relative protein concentration.

Statistical analysis. Results are expressed as the mean \pm standard deviation, and differences among the groups were analyzed using univariate analysis of variance and the least significant difference test. SPSS 13.0 software (SPSS, Inc., Chicago, IL, USA) was used for all the statistical analyses, and $\mathrm{P}<0.05$ was considered to indicate a statistically significant difference.

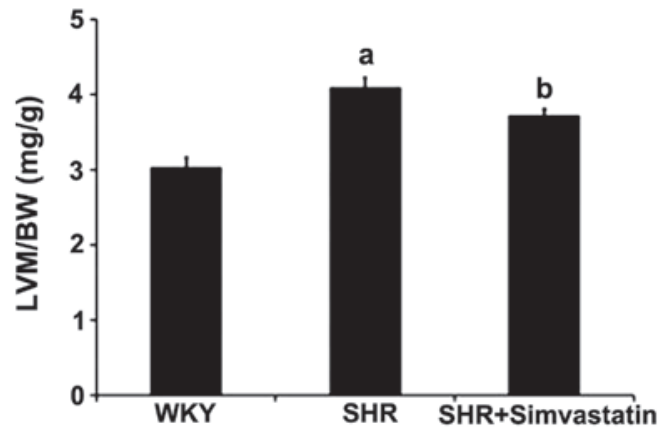

Figure 1. Simvastatin reduces cardiomyocyte hypertrophy in SHRs, as indicated by a higher LVW/BW ratio. ${ }^{\mathrm{a}} \mathrm{P}<0.01$, vs. WKY; ${ }^{\mathrm{b}} \mathrm{P}<0.01$, vs. SHR. SHR, spontaneously hypertensive rats; WKY, Wistar-Kyoto; LVM, left ventricular mass; BM, body mass.

\section{Results}

Simvastatin administration prevents cardiomyocyte hypertrophy in SHRs. The LVM/BW ratio has been used to indicate cardiomyocyte hypertrophy in certain in vivo experiments. In the control (WKY) group, the LVM/BW ratio was $3.04 \pm 0.12 \mathrm{mg} / \mathrm{g}$, while in SHRs, the ratio was determined to be $4.10 \pm 0.13 \mathrm{mg} / \mathrm{g}$. Treatment with simvastatin was shown to reduce the extent of cardiomyocyte hypertrophy in the SHRs, with simvastatin treatment decreasing the LVM/BW ratio to $3.73 \pm 0.08 \mathrm{mg} / \mathrm{g}$ (Fig. 1).

Simvastatin inhibits PKB expression, but increases PTEN expression in SHRs. In order to identify the pathway affected by simvastatin with regard to cardiomyocyte hypertrophy, the protein expression levels of PKB and PTEN were investigated in the study. In the SHR group, the PKB protein expression levels were significantly greater when compared with that in the WKY group $(\mathrm{P}<0.01)$, while the level in the simvastatin + SHR group was significantly lower when compared with the SHR group $(\mathrm{P}<0.01)$. By contrast, the protein expression level of PTEN in the SHR group was significantly lower when compared with that in the WKY group $(\mathrm{P}<0.01)$, while expression levels were significantly increased in the simvastatin treatment group, as compared with that of the SHR group ( $\mathrm{P}<0.01$; Fig. 2A-C).

Effects of simvastatin on the surface area and 3 H-leucine incorporation in cultured cardiomyocytes. Following treatment with $15 \%$ neonatal bovine serum for $24 \mathrm{~h}$, the cardiomyocyte surface area was $1,611.16 \pm 160.75 \mu \mathrm{m}^{2}$, which was significantly higher when compared with that of the serum-free control group (538.04 $\left.\pm 118.60 \mu \mathrm{m}^{2} ; \mathrm{P}<0.01\right)$. Following treatment with different concentrations of simvastatin together with $15 \%$ fetal bovine serum, the cardiomyocyte surface area was determined to be significantly lower in the $10^{-5}$ and $10^{-6} \mathrm{~mol} / \mathrm{l}$ simvastatin intervention groups $\left(799.84 \pm 167.70\right.$ and $1,076.88 \pm 199.28 \mu \mathrm{m}^{2}$, respectively), as compared with the serum group $(\mathrm{P}<0.01)$. However, in the $10^{-7}$ and $10^{-8} \mathrm{~mol} / \mathrm{l}$ simvastatin intervention groups, the cardiomyocyte surface areas $(1,529.32 \pm 212.83$ and $1,606.84 \pm 220.81 \mu \mathrm{m}^{2}$, respectively) were not significantly different when compared with that of the serum group $(\mathrm{P}>0.05$; Fig. 3A). 
A

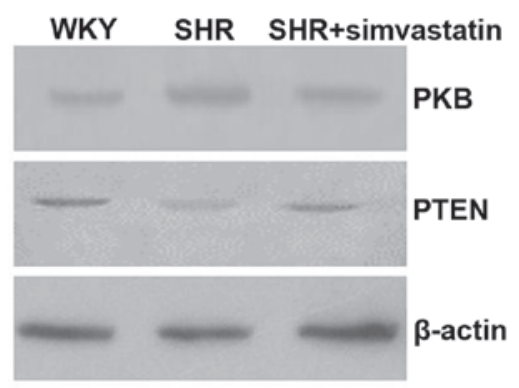

B

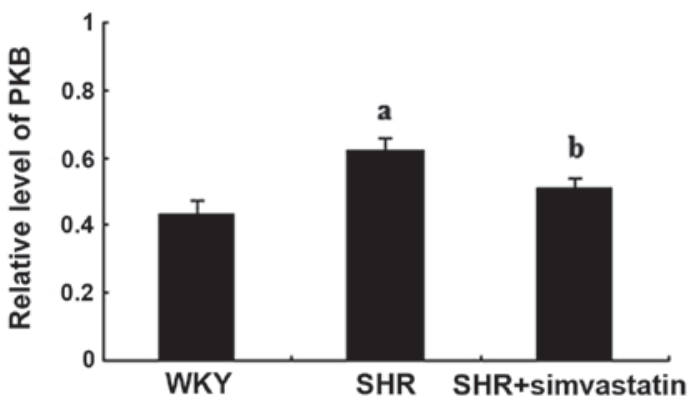

C

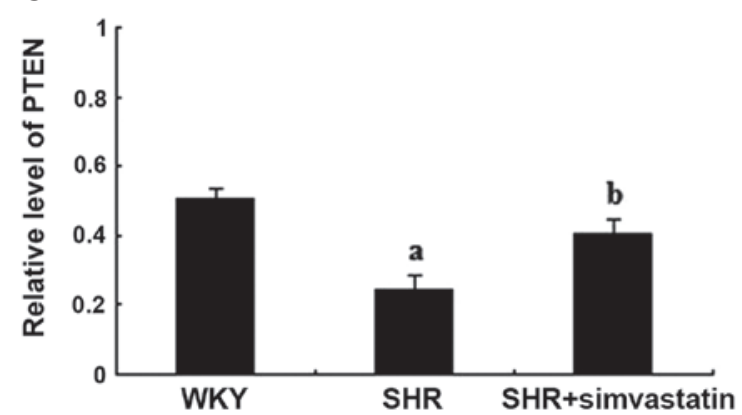

Figure 2. (A) Representative western blot showing the expression levels of PKB and PTEN in the three groups. Simvastatin was found to (B) inhibit PKB expression, but (C) increase PTEN expression in the SHR. ${ }^{a} \mathrm{P}<0.01$, vs. WKY; ${ }^{b} \mathrm{P}<0.01$, vs. SHR. SHR, spontaneously hypertensive rats; WKY, Wistar-Kyoto; PKB, protein kinase B; PTEN, phosphatase and tensin homolog.

A

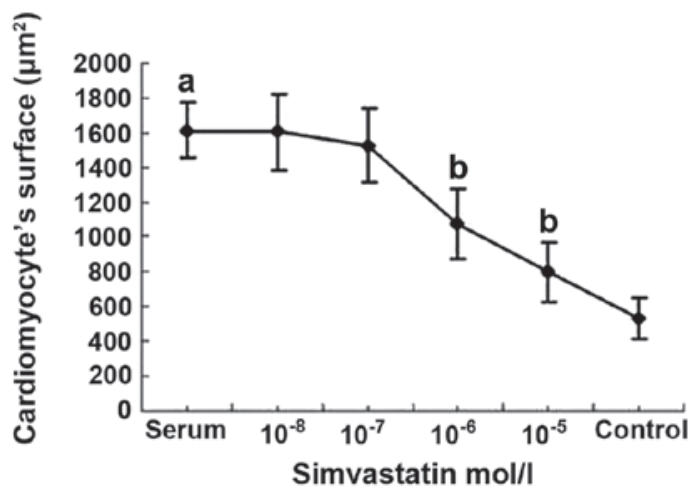

B

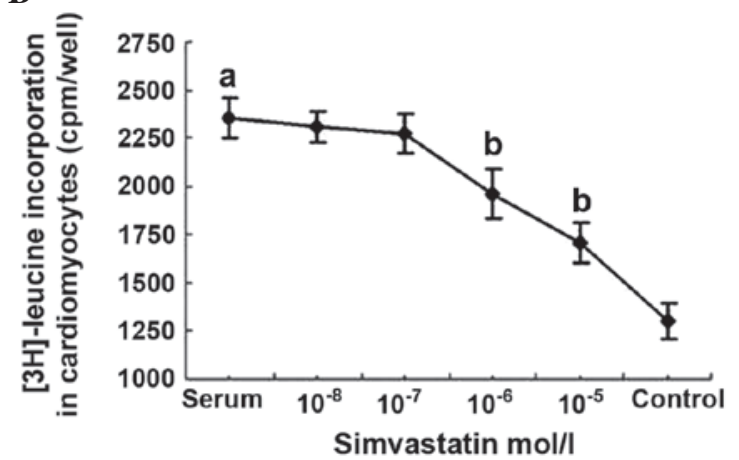

Figure 3. Simvastatin treatment blocks the serum-induced increase in (A) cardiomyocyte surface area and (B) 3H-leucine incorporation rate in cultured cardiomyocytes. ${ }^{\mathrm{a}} \mathrm{P}<0.01$, vs control group; ${ }^{\mathrm{b}} \mathrm{P}<0.01$, vs serum group.

The $3 \mathrm{H}$-leucine incorporation rate in the $15 \%$ neonatal bovine serum group $(2,360 \pm 106 \mathrm{cpm} /$ well $)$ was significantly greater when compared with that in the serum-free group $(1,305 \pm 92 \mathrm{cpm} /$ well; $\mathrm{P}<0.01)$. The incorporation rates were $1,707 \pm 101$ and $1,962 \pm 125 \mathrm{cpm} /$ well in the $10^{-5}$ and $10^{-6} \mathrm{~mol} / 1$ simvastatin intervention groups, respectively, and these values were significantly lower when compared with the serum group $(\mathrm{P}<0.01)$. In the $10^{-7}$ and $10^{-8} \mathrm{~mol} / \mathrm{l}$ simvastatin intervention groups, the incorporation rates were $2,280 \pm 105$ and $2,311 \pm 80 \mathrm{cpm} /$ well, respectively; however, these values were not significantly different when compared with that of the serum group ( $\mathrm{P}>0.05$; Fig. 3B).

Effects of simvastatin on PKB and PTEN expression in the cultured cardiomyocytes. PKB protein expression levels were significantly higher in the serum group when compared with the control group $(\mathrm{P}<0.01)$. Furthermore, the PKB protein expression levels were significantly lower in the $10^{-5}$ and $10^{-6} \mathrm{~mol} / \mathrm{l}$ simvastatin intervention groups when compared with the serum group $(\mathrm{P}<0.01)$. However, there were no statistically significant differences in the levels when comparing the $10^{-7}$ and $10^{-8}$ simvastatin intervention groups with the serum group $(\mathrm{P}>0.05)$. By contrast, simvastatin was found to increase the PTEN protein expression levels in a concentration-dependent manner. The protein expression levels of PTEN in the $10^{-5}, 10^{-6}$ and $10^{-7} \mathrm{~mol} / 1$ simvastatin + serum groups were significantly higher when compared with the serum group (all $\mathrm{P}<0.05$ ); however, the expression levels were all lower when compared with the control group (all $\mathrm{P}<0.01$; Fig. 4A-C).

PTEN antisense oligodeoxynucleotides decrease the level of PTEN and inhibit the effect of simvastatin on cardiomyocytes. Inhibition of the biological effects of simvastatin 
A

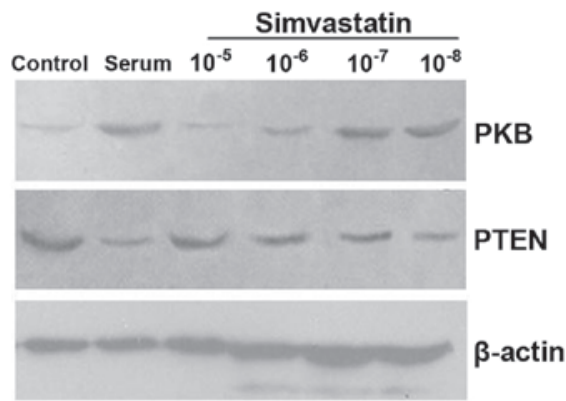

C
B

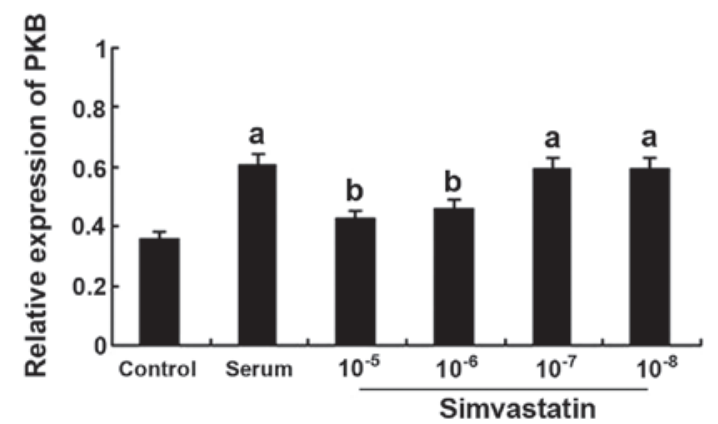

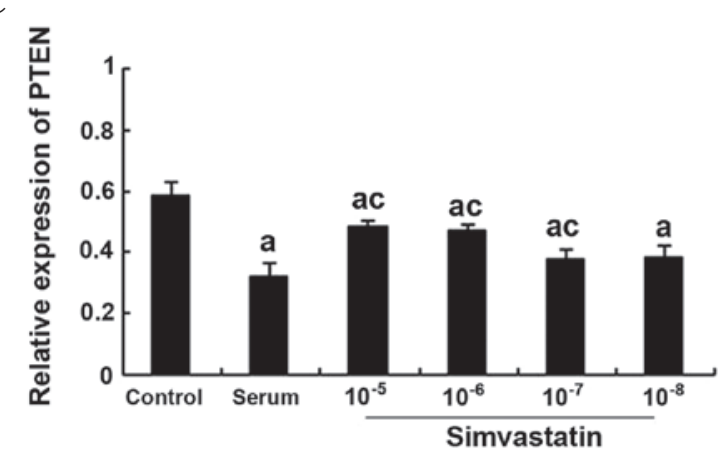

Figure 4. Effects of simvastatin on PKB and PTEN expression in cultured cardiomyocytes. (A) Representative western blot, and quantitative determination of (B) PKB and (C) PTEN protein expression levels in the control, serum, and $10^{-5}, 10^{-6}, 10^{-7}$ and $10^{-8} \mathrm{~mol} / 1$ simvastatin intervention groups. ${ }^{\mathrm{a}} \mathrm{P}<0.01$, vs. control group; ${ }^{\mathrm{P}} \mathrm{P}<0.01$, vs. serum group; ${ }^{\mathrm{C}} \mathrm{P}<0.05$, vs. serum group. $\mathrm{PKB}$, protein kinase $\mathrm{B}$; $\mathrm{PTEN}$, phosphatase and tensin homolog.

A

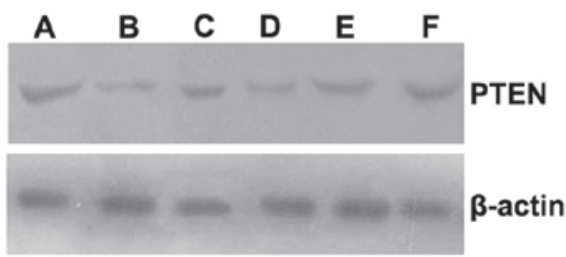

C

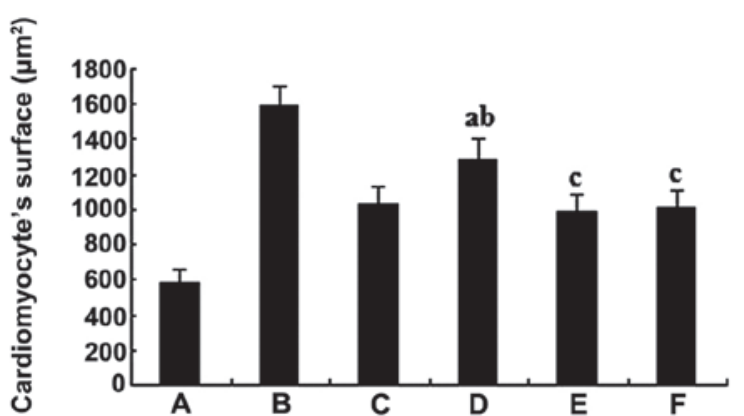

B

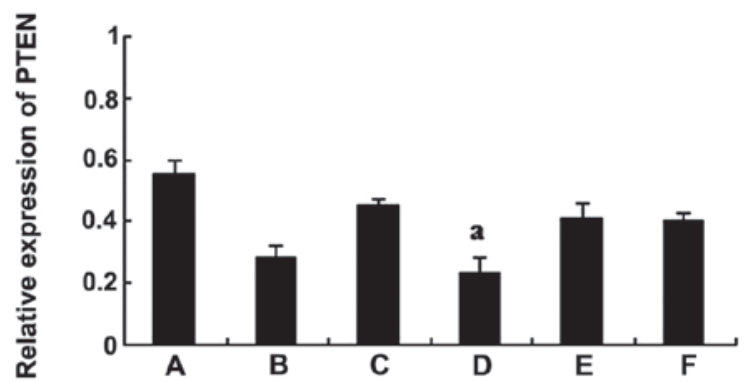

D

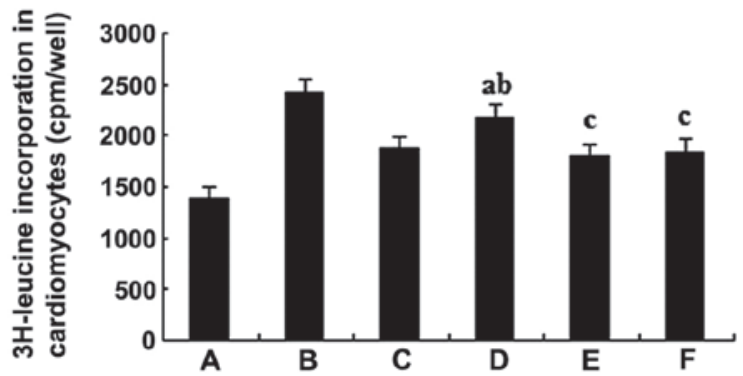

Figure 5. PTEN antisense oligodeoxynucleotides decrease the level of PTEN protein expression and inhibit the effects of simvastatin on cardiomyocytes. (A) Representative western blot and (B) quantitative determination of PTEN protein expression levels. (C) Cardiomyocyte surface area and (D) $3 \mathrm{H}-\mathrm{leucine}$ incorporation rate of the various groups. ${ }^{a} \mathrm{P}<0.01$, vs. $10^{-6} \mathrm{~mol} / 1$ simvastatin group; ${ }^{b} \mathrm{P}<0.01$, vs. serum group; ${ }^{\mathrm{c}} \mathrm{P}>0.05$, vs. $10^{-6} \mathrm{~mol} / 1$ simvastatin group. $\mathrm{PTEN}$, phosphatase and tensin homolog; A, control group; B, serum group; C, $10^{-6} \mathrm{~mol} / 1$ simvastatin group; $\mathrm{D}, 10^{-6} \mathrm{~mol} / \mathrm{l}$ simvastatin + PTEN antisense oligodeoxynucleotides group; E, $10^{-6} \mathrm{~mol} / 1$ simvastatin + PTEN sense oligodeoxynucleotides group; F, $10^{-6} \mathrm{~mol} / 1$ simvastatin + PTEN mismatch oligodeoxynucleotides group.

on the cardiomyocytes was observed following treatment with PTEN antisense oligodeoxynucleotides. The protein expression levels of PTEN in the PTEN antisense oligodeoxynucleotides group were significantly lower compared with those in the simvastatin group $(\mathrm{P}<0.01)$; however, there were no statistically significant differences when comparing the sense or mismatch groups with the simvastatin group (P>0.05; Fig. 5A and B). Accordingly, the cardiomyocyte 
surface area (Fig. 5C) and the $3 \mathrm{H}$-leucine incorporation rate (Fig. 5D) in the $10^{-6} \mathrm{~mol} / \mathrm{l}$ simvastatin $+5 \times 10^{-6} \mathrm{~mol} / \mathrm{l}$ PTEN antisense oligodeoxynucleotides group were significantly greater compared with the values observed in the simvastatin single treatment group $(\mathrm{P}<0.01)$. However, the values were lower compared with those of the serum group $(\mathrm{P}<0.01)$. By contrast, there were no statistically significant differences in the protein expression levels when comparing the PTEN sense and mismatch oligodeoxynucleotides group with the simvastatin group $(\mathrm{P}>0.05)$.

\section{Discussion}

The salient finding of the present study was that simvastatin reduces cardiomyocyte hypertrophy by increasing PTEN expression. In the current study, simvastatin administration was demonstrated to prevent rat cardiomyocyte hypertrophy and inhibit PKB expression in vivo and in vitro. By contrast, simvastatin was shown to significantly increase PTEN expression in the SHRs and cultured cardiomyocytes. In addition, treatment with PTEN antisense oligodeoxynucleotides significantly inhibited the expression of PTEN and blocked the effect of simvastatin on cardiomyocytes. These results demonstrate possible molecular pathways underlying the effects of simvastatin on the hypertrophy of cardiomyocytes, and indicate that simvastatin may be an effective pharmacological compound against cardiomyocyte hypertrophy.

Statins are well accepted to not only have satisfactory lipid regulatory effects, but also inhibit the proliferation of vascular smooth muscle cells and cardiac fibroblasts $(14,15)$. Statin administration is associated with a decreased incidence of cardiovascular events and mortality in patients with cardiovascular risk. However, the effects of statins on cardiomyocytes and the specific molecular mechanisms underlying their action remain uncertain.

A previous study demonstrated that fetal bovine serum containing endothelin I, angiotensin II and fibroblast growth factors is able to induce the hypertrophy of cardiomyocytes (16). In the present study, simvastatin was shown to inhibit the serum-induced increase in cell surface area and the $3 \mathrm{H}$-leucine incorporation rate in cardiomyocytes, while significantly decreasing the PKB expression levels in a concentration-dependent manner. Leucine is a precursor of protein synthesis, and the $3 \mathrm{H}$-leucine incorporation assay is widely used for the detection of protein synthesis in cells. The results of the present study indicated that simvastatin inhibited the hypertrophy and protein synthesis of cardiomyocytes, which may be significant in alleviating hypertension-induced $\mathrm{LVH}$, improving heart function and delaying the development of cardiac hypertrophy to heart failure.

In addition to the classical function of statins on lipoproteins, there is accumulating evidence that statins also reduce the level of cellular isoprenoid intermediates and inhibit the isoprenylation of small molecular weight G-proteins of Ras/Rho, to subsequently exert protective effects on the cardiovascular system (2). Statins play a role in cell proliferation and hypertrophy through mediating the membrane translocation of Ras protein, and the MAPK and PI3K/PKB signaling pathways are involved in the biological functions of Ras protein (3). In addition, the PI3K/PKB pathway has been found to be closely involved in the proliferation, hypertrophy, apoptosis and survival process of cells (4). Nevertheless, it is unclear how the pathway is involved in the statin-modulated hypertrophy process of cardiomyocytes. In the present study, simvastatin was demonstrated to reverse the serum-induced hypertrophy of cardiomyocytes, as well as decrease the protein expression levels of PKB. Therefore, the results indicate that PKB is an important signal transmission molecule involved in the hypertrophy of cardiomyocytes. Simvastatin is able to downregulate PKB expression, modulate the activity of downstream kinases and transcription factors, and decrease the expression of protein synthesis-associated genes, in order to inhibit the hypertrophy of cardiomyocytes.

PTEN expression levels may be involved in myocardial hypertrophy, which can be inferred since simvastatin was shown to elevate the PTEN expression level. External prohypertrophic factors downregulate PTEN expression in cardiomyocytes, which subsequently activates downstream kinases and transcription factors through the PI3K/PKB pathway, thus motivating myocardium hypertrophy. This may be one of the molecular mechanism through which simvastatin inhibits the hypertrophy of cardiomyocytes and reverses LVH. The biological effects of simvastatin on cardiomyocytes may be partly inhibited by intervention with PTEN antisense oligodeoxynucleotides. In the present study, application of PTEN antisense oligodeoxynucleotides reduced the simvastatin-induced increase in PTEN expression, and also decreased the cardiomyocyte surface area and $3 \mathrm{H}$-leucine incorporation rate, indicating that PTEN is involved in the effect of simvastatin on the hypertrophy of cardiomyocytes.

In conclusion, the results of the present study indicate that simvastatin is able to reverse cardiomyocyte hypertrophy induced by fetal bovine serum, possibly through increasing PTEN expression, thereby inhibiting the PI3K/PKB pathway. Therefore, the present study provides a novel theoretical and experimental basis for the alleviation of LVH at the cellular and molecular levels, and, furthermore, offers new insights for the prevention of hypertension-induced LVH.

\section{References}

1. Zheng $\mathrm{H}$ and Lu GM: Reduction of prohibitin expression contributes to left ventricular hypertrophy via enhancement of mitochondrial reactive oxygen species formation in spontaneous hypertensive rats. Free Radic Res 49: 164-174, 2015.

2. Koh KK, Sakuma I and Quon MJ: Differential metabolic effects of distinct statins. Atherosclerosis 215: 1-8, 2011.

3. Sugden PH: Ras, Akt, and mechanotransduction in the cardiac myocyte. Circ Res 93: 1179-1192, 2003.

4. Cantley LC: The phosphoinositide 3-kinase pathway. Science 296: 1655-1657, 2002.

5. Paez J and Sellers WR: PI3K/PTEN/AKT pathway. A critical mediator of oncogenic signaling. Cancer Treat Res 115: 145-167, 2003.

6. Shiojima I and Walsh K: Regulation of cardiac growth and coronary angiogenesis by the Akt/PKB signaling pathway. Genes Dev 20: 3347-3365, 2006.

7. van Berlo JH, Maillet M and Molkentin JD. Signaling effectors underlying pathologic growth and remodeling of the heart. J Clin Invest 123: 37-45, 2013.

8. Stuenaes JT, Bolling A, Ingvaldsen A, et al: Beta-adrenoceptor stimulation potentiates insulin-stimulated PKB phosphorylation in rat cardiomyocytes via cAMP and PKA. Br J Pharmacol 160: 116-129, 2010. 
9. Maire CL, Ramkissoon S, Hayashi M, Haidar S, Ramkissoon L, DiTomaso E and Ligon KL: Pten loss in Olig2 expressing neura progenitor cells and oligodendrocytes leads to interneuron dysplasia and leukodystrophy. Stem Cells 32: 313-326, 2014.

10. Crackower MA, Oudit GY, Kozieradzki I, et al: Regulation of myocardial contractility and cell size by distinct PI3K-PTEN signaling pathways. Cell 110: 737-749, 2002.

11. Zhang J, Cheng X, Liao YH, et al: Simvastatin regulates myocardial cytokine expression and improves ventricular remodeling in rats after acute myocardial infarction. Cardiovasc Drugs Ther 19: 13-21, 2005.

12. Simpson P, McGrath A and Savion S: Myocyte hypertrophy in neonatal rat heart cultures and its regulation by serum and by catecholamines. Circ Res 51: 787-801, 1982.
13. Choi YS, Dusting GJ, Stubbs S, et al. Differentiation of human adipose-derived stem cells into beating cardiomyocytes. J Cell Mol Med 14: 878-889, 2010.

14. Clunn GF, Sever PS and Hughes AD: Calcium channel regulation in vascular smooth muscle cells: Synergistic effects of statins and calcium channel blockers. Int J Cardiol 139: 2-6, 2010.

15. Wang Q, Cui W, Zhang HL, Hu HJ, Zhang YN, Liu DM and Liu J: Atorvastatin suppresses aldosterone-induced neonatal rat cardiac fibroblast proliferation by inhibiting ERK $1 / 2$ in the genomic pathway. J Cardiovasc Pharmacol 61: 520-527, 2013.

16. Santalucía T, Christmann M, Yacoub MH and Brand NJ: Hypertrophic agonists induce the binding of c-Fos to an AP-1 site in cardiac myocytes: Implications for the expression of GLUT1. Cardiovasc Res 59: 639-648, 2003. 